\title{
Beyond the charge radius: The information content of the fourth radial moment
}

\author{
P.-G. Reinhard $\odot,{ }^{1}$ W. Nazarewicz $\odot,{ }^{2}$ and R. F. Garcia Ruiz ${ }^{3,4}$ \\ ${ }^{1}$ Institut für Theoretische Physik, Universität Erlangen, D-91054 Erlangen, Germany \\ ${ }^{2}$ Department of Physics and Astronomy and FRIB Laboratory, Michigan State University, East Lansing, Michigan 48824, USA \\ ${ }^{3}$ Physics Department, CERN, CH-1211 Geneva 23, Switzerland \\ ${ }^{4}$ Massachusetts Institute of Technology, Cambridge, Massachusetts 02139, USA
}

(Received 24 September 2019; revised manuscript received 19 November 2019; accepted 23 January 2020; published 6 February 2020)

\begin{abstract}
Measurements of atomic transitions in different isotopes offer key information on the nuclear charge radius. The anticipated high-precision experimental techniques, augmented by atomic calculations, will soon enable extraction of the higher order radial moments of the charge density distribution. To assess the value of such measurements for nuclear structure research, we study the information content of the fourth radial moment $\left\langle r^{4}\right\rangle$ by means of nuclear density functional theory and a multiple correlation analysis. We show that $\left\langle r^{4}\right\rangle$ can be directly related to the surface thickness of nuclear density, a fundamental property of the atomic nucleus that is difficult to obtain for radioactive systems. Precise knowledge of these radial moments is essential to establish reliable constraints on the existence of new forces from precision isotope shift measurements.
\end{abstract}

DOI: 10.1103/PhysRevC.101.021301

Introduction. A precise knowledge of the electron-nucleus interaction in atoms can provide access to physical phenomena relevant to a wide range of energy scales. High-precision measurements of atomic transitions, for example, offer complementary information to our understanding of the atomic nucleus, the study of fundamental symmetries, and the search for new physics beyond the standard model of particle physics [1-4].

Varying the number of neutrons induces changes in the charge density distribution along the isotopic chain, causing tiny perturbations in the energies of their atomic electrons, known as isotope shifts. Measurements of the corresponding frequencies, typically of the order of $\mathrm{MHz}$, allow changes in the root-mean-squared ( $\mathrm{rms}$ ) nuclear charge radii to be extracted [5,6]. Extending these measurements for isotopes away from stability is of marked and growing interest for low-energy nuclear physics, as the data on the nuclear size are essential for our understanding of the nuclear many-body problem [5,7-10]. In recent years, the interest in precision isotope shift measurements has increased significantly. Performing measurements across long isotope chains that are readily available at state-of-the-art radioactive ion beam facilities has the potential to constrain the existence of new forces and hypothetical particles with unprecedented sensitivity [2-4,1114]. This has motivated the rapid progress of experimental techniques which are continuously pushing the frontiers of precision measurements. Quantum logic detection schemes

Published by the American Physical Society under the terms of the Creative Commons Attribution 4.0 International license. Further distribution of this work must maintain attribution to the author(s) and the published article's title, journal citation, and DOI. have achieved sub-MHz precision [15], and recent developments such as spin squeezing [16] and quantum entanglement [17] are now able to reach sub-Hz precision. This level of precision offers sensitivity not only would allow us to explore new physics but would also provide access to nuclear observables that have so far been elusive, such as the higher order radial moment $\left\langle r^{4}\right\rangle[18,19]$ and the nuclear dipole polarizability [13]. Precise knowledge of these nuclear properties will open up exciting opportunities in nuclear structure research and hence is essential to establish reliable constraints in the exploration of new physics $[13,14]$. In addition to the progress of high-precision experiments, the continued development of atomic and nuclear theory has played a crucial role in extracting nuclear structure and fundamental physics observables from measurements [18-20].

The isotope shift, $\Delta v_{i}$, between an isotope with mass, $A$, and an isotope $A^{\prime}$, can be expressed by a product of nuclear and atomic factors as

$$
\Delta v_{i}^{A A^{\prime}}=K_{\mathrm{MS}, i} \frac{A-A^{\prime}}{A A^{\prime}}+\sum_{k} F_{i, k} \delta\left\langle r^{2 k}\right\rangle^{A A^{\prime}},
$$

where $\delta\left\langle r^{2 k}\right\rangle^{A A^{\prime}}$ is the difference between the nuclear radial moments of order $2 k$. The atomic part is factorized in the constants $K_{\mathrm{MS}, i}$ and $F_{i}$, referred to as the mass shift and the field shift, respectively. Assuming a negligible contribution from $k>1$ moments, isotope shifts from different atomic transition $i$ and $j, \Delta v_{i}^{A A^{\prime}}$ versus $\Delta v_{j}^{A A^{\prime}}$, should follow a linear relation known as the King plot [21]. The nonlinearity of the King plot can be due to the contribution from $k>1$ moments. It can also indicate the presence of new phenomena [2-4,1113]. Therefore, the estimation of the effect higher order terms is important to provide bounds on physics beyond the standard model. As discussed in Refs. [18,19], taking advantage of the improved experimental precision and atomic calculations with 
well-controlled uncertainty quantification for atomic states will enable us to extract highly accurate atomic line field shifts and higher order radial moments. To assess the impact of the anticipated data on our understanding of atomic nuclei, in this Rapid Communication, we employ density functional theory to study the $k=2$ moment $\left\langle r^{4}\right\rangle$ of nuclear charge distribution.

Nuclear charge distribution characteristics. The gross features of the nuclear charge distribution $\rho(\boldsymbol{r})$ and its charge form factor $F(\boldsymbol{q})$ can be described by form parameters: radial moments

$$
r_{n} \equiv \sqrt[n]{\left\langle r^{n}\right\rangle}=\left(\frac{\int d^{3} r r^{n} \rho(\boldsymbol{r})}{\int d^{3} r \rho(\boldsymbol{r})}\right)^{1 / n},
$$

diffraction radius $R$, and surface thickness $\sigma$. The latter characterizes the density diffuseness around the nuclear surface. The rms charge radius is given by the second moment $r_{2}$. The diffraction radius is determined from the first zero of the form factor $F(q)$ (the diffraction minimum). It represents a box-equivalent radius. The surface thickness is determined from the height of the first maximum of $F(q)$. The relations between spatial geometrical parameters and the form factor are provided by the Helm model [22], which represents the nuclear density profile by a folding of a box distributions (having radius $R$ ) with a Gaussian of width $\sigma$. For details, see Refs. [23-26] and the Supplemental Material [27]. Within the Helm model, $r_{2}$ can be expressed in terms of $R$, and $\sigma$ : $r_{2}^{(\mathrm{H})}=\sqrt{\frac{3}{5} R^{2}+3 \sigma^{2}}$. In practice, this relation is not exactly fulfilled and the deviation characterizes the halo of the charge distribution [28-30]:

$$
h=r_{2}-r_{2}^{(\mathrm{H})}
$$

In practice, the halo is a small positive quantity [28]. Diffused charge distributions associated with loosely bound protons in proton-rich isotopes produce appreciable values of $h$. To give an idea about the typical values and trends, we show in Fig. 1 the charge density form parameters for the chain of $\mathrm{Sn}$ isotopes calculated with two nuclear energy density functionals: the Skyrme parametrization SV-min [31] and the Fayans functional Fy $(\Delta r, \mathrm{HFB})[10,27,32]$. (For the treatment of pairing in natural orbital representation in $\mathrm{Fy}(\Delta r, \mathrm{HFB})$ see Refs. [33-35].) Both functionals have been optimized with respect to the pool of empirical data from [31], with some additional charge radii data used in the optimization of $\mathrm{Fy}(\Delta r, \mathrm{HFB})$. The fits allow us also to deduce the statistical uncertainties on the predicted observables by standard linear regression methods [36]. The uncertainties are consistent with the adopted errors for the observables, which are $\pm 0.04 \mathrm{fm}$ for $R$ and $\sigma$ and $\pm 0.02 \mathrm{fm}$ for $r_{2}$. These errors do not contain the experimental uncertainties but reflect the capability of the model to reproduce observables. A measurement is expected to provide new information if the experimental uncertainty is safely below the model error. The values of the form parameters show the expected trends [28]. Namely, the proton radii shrink systematically with increasing neutron number because of the increasing proton binding. The pronounced kink at the $N=82$ shell closure seen in all form parameters is due to reduced neutron pairing [9]. The two density functionals used deliver similar results in the domain of

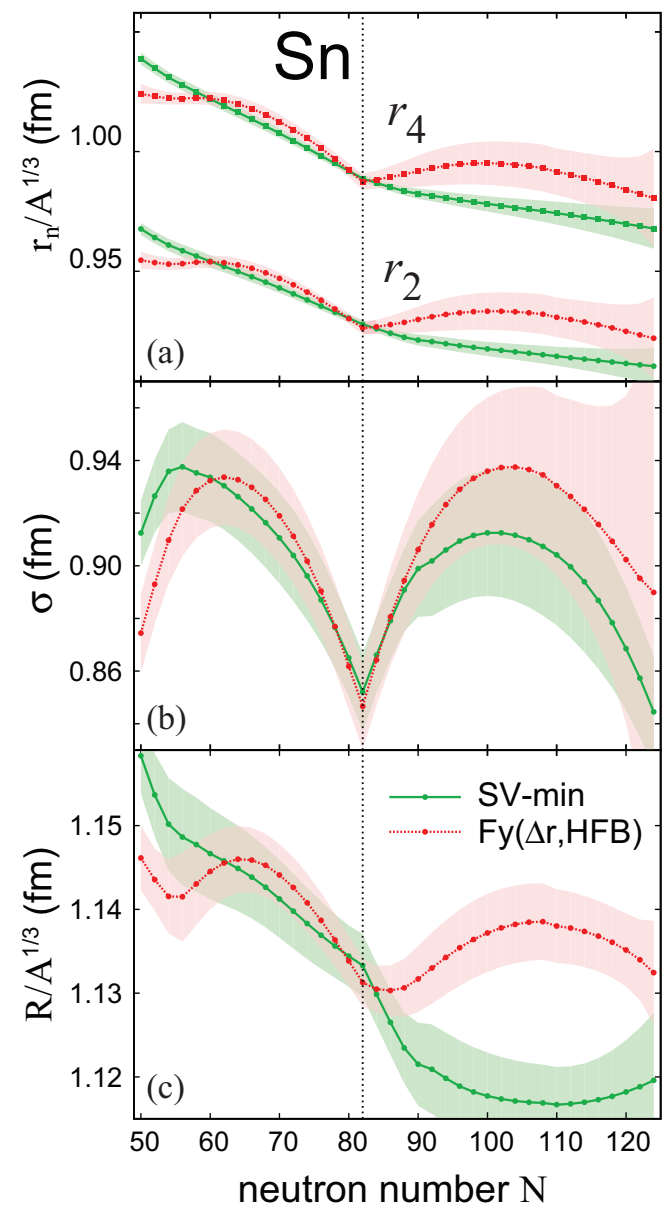

FIG. 1. Form parameters along the Sn chain $(Z=50)$ computed with energy density functionals SV-min and $\mathrm{Fy}(\Delta r)$ together with their statistical uncertainties. Shown are radial moments $r_{2}$ and $r_{4}$ (a), surface thickness $\sigma(\mathrm{b})$, and diffraction radii $R(\mathrm{c})$. To better visualize the local trends, radial moments and diffraction radii are scaled by $A^{1 / 3}$. The magic neutron number $N=82$ is marked.

well-bound nuclei while developing slight differences at exotic proton-rich nuclei close to $N=50$ and neutron-rich nuclei with $N>82$. This is entirely anticipated: Form parameters of stable nuclei, being part of the optimization data pool, are bound to be well reproduced. The differences between $\mathrm{SV}$-min and $\mathrm{Fy}(\Delta r, \mathrm{HFB})$ in neutron-rich isotopes are almost exclusively generated by the gradient-pairing term of the Fayans functional. At the neutron-rich side, the difference for the diffraction radii $R$ can amount up to about $0.1 \mathrm{fm}$, clearly above the error bars.

Statistical analysis. The information content of $r_{4}$ is evaluated using standard statistical correlation analysis as in Refs. [9,37]. The question we ask is to what extent $r_{4}$ is already determined by the other form parameters and, vice versa, to what extent information on $r_{4}$ improves our knowledge of $R$ and $\sigma$. The answer can be quantified in terms of statistical correlations. Those between two observables $A$ and $B$ are described by the coefficient of determination $\operatorname{CoD}(A, B)$ deduced from the covariance measure. Furthermore, we inspect multiple correlation coefficients $\operatorname{MCC}\left(A_{1}, \ldots, A_{n} ; B\right)$, 


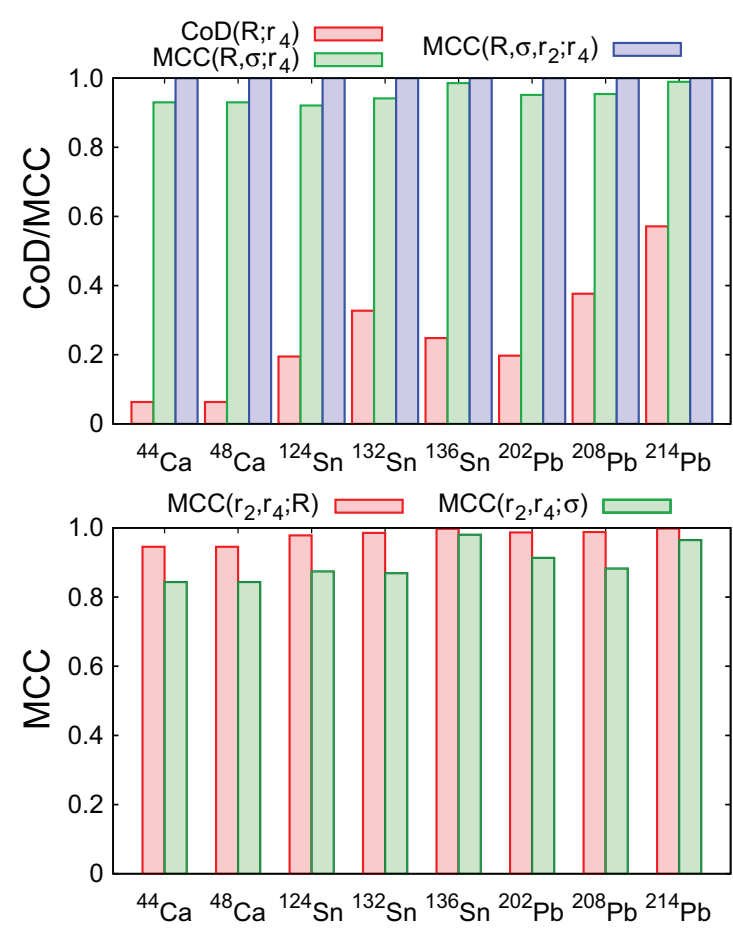

FIG. 2. Top: CoD and MCC coefficients between the standard form parameters and $r_{4}$ for a selection of spherical nuclei. Bottom: information content of $r_{2}$ and $r_{4}$ in terms of MCCs between $r$ and $r_{4}$ with the form parameters $R$ or $\sigma$.

which characterize the correlations between a group of observables $A_{1}, \ldots, A_{n}$ with $B$ [38]. The MCC is reduced to the $\mathrm{CoD}$ if $n=1$. The CoDs and MCCs range from 0 to 1 , where 0 implies that the observable $B$ is uncorrelated with the group of observables $A_{i}$ and the value of 1 means full correlation.

The results of our correlation analysis are shown in Fig. 2. The upper panel explores the prediction of $r_{4}$ for known $R$, $\sigma$, and $r_{2}$ for a set of spherical nuclei, which have very small halo. The diffraction radius alone has little predictive value for $r_{4}$. This is not surprising as $R$ carries no information on surface diffuseness, which strongly impacts the fourth radial moment. The combination of $R$ and $\sigma$ provides a very good 95\% estimate of $r_{4}$. Finally, the group of $R$ and $\sigma$, and $r_{2}$ (or h) manages to determine $r_{4}$ fully.

The lower panel of Fig. 2 explores whether a simultaneous measurement of $r_{2}$ and $r_{4}$ can determine $R$ or $\sigma$. The MCCs show that the diffraction radius is indeed very well determined by $r_{2}$ and $r_{4}$, especially for heavy nuclei. The surface thickness is also well predicted, although not as perfectly as $R$, typically at a $90 \%$ level.

Helm model analysis. Statistical analysis, although well defined and extremely useful, remains largely a black box. To gain more physics insights, we study interrelations between the form parameters as mediated by the Helm model, which is commonly used to inform nuclear energy density functionals about experimental charge form factor. We checked some alternative models with more realistic exponential asymptotics [25], and after finding no significant differences in the context of our work, we decided to stick to the Helm model as the simplest one. Given $r_{2}$ and $r_{4}$, we deduce closed approximate

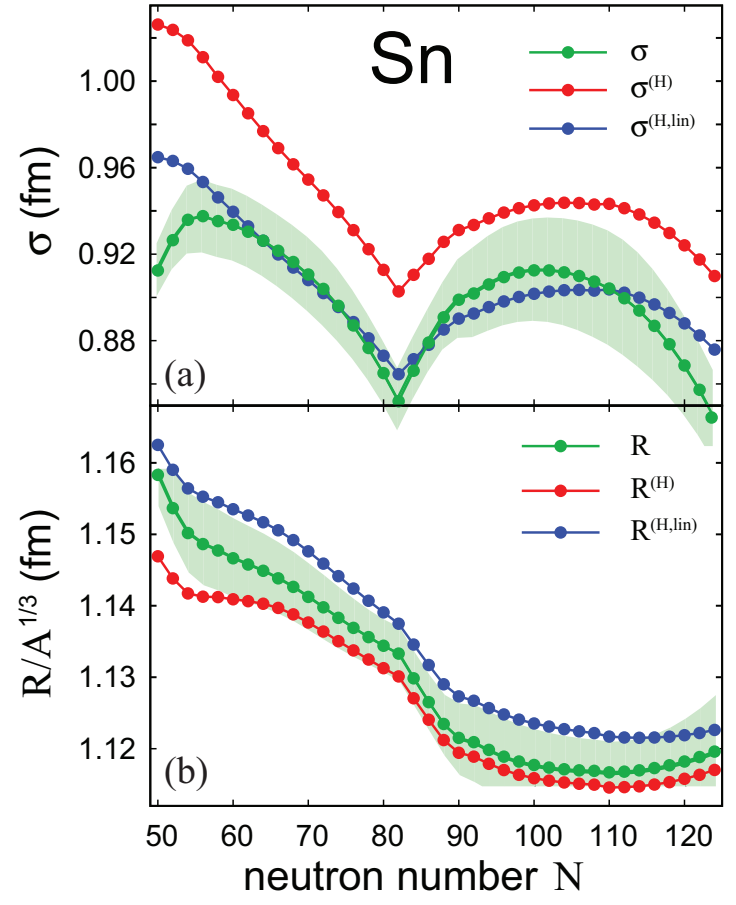

FIG. 3. Helm-model SV-min predictions (4)-(7) for $\sigma$ (top) and $R$ (bottom) for the chain of $\mathrm{Sn}$ isotopes. The values of $\sigma$ and $R$ extracted from the charge density form factor are also shown together with their uncertainties.

expression for $\sigma$ and $R$, again denoted by an upper index (H) to distinguish them from the exact values; see Ref. [27] for details. For more compact expressions, we introduce the rescaled geometric radii as $R_{n}^{(\mathrm{g})}=\sqrt[n]{\frac{n+3}{3} r_{n}^{n}}$ [28].

In terms of $R_{2}^{(\mathrm{g})}$ and $R_{4}^{(\mathrm{g})}$ the Helm-model values of diffraction radius and surface thickness are [27]

$$
\begin{aligned}
R^{(\mathrm{H})} & =\sqrt[4]{\frac{7}{2} R_{2}^{(\mathrm{g})^{4}}-\frac{5}{2} R_{4}^{(\mathrm{g})^{4}}}, \\
\sigma^{(\mathrm{H})} & =\sqrt{\frac{1}{5} R_{2}^{(\mathrm{g})^{2}}\left(1-\sqrt{\left.1-\frac{5}{2} \frac{R_{4}^{(\mathrm{g})^{4}}-R_{2}^{(\mathrm{g})^{4}}}{R_{2}^{(\mathrm{g})^{4}}}\right)} .\right.} .
\end{aligned}
$$

Another set of useful relations can be obtained by noticing that $\sigma^{2} / R^{2}$ is a small parameter, which is around 0.02-0.03 (see Fig. 1). By linearizing the above relations with respect to $\sigma^{2} / R^{2}$, one obtains the following approximate relations:

$$
\begin{aligned}
R^{(\mathrm{H}), \text { lin }} & \approx R_{2}^{(\mathrm{g})}+\frac{7}{2}\left(R_{4}^{(\mathrm{g})}-R_{2}^{(\mathrm{g})}\right), \\
\sigma^{(\mathrm{H}), \text { lin }} & \approx \sqrt{\left(R_{4}^{(\mathrm{g})}-R_{2}^{(\mathrm{g})}\right) R_{2}^{(\mathrm{g})}}, \\
\frac{R_{4}^{(\mathrm{g})}-R_{2}^{(\mathrm{g})}}{R_{2}^{(\mathrm{g})}} & \approx\left(\frac{\sigma^{(\mathrm{H}), \operatorname{lin}}}{R^{(\mathrm{H}), \text { lin }}}\right)^{2} .
\end{aligned}
$$

Figure 3 compares the Helm model values of $R$ and $\sigma$ given by Eqs. (4)-(7) to the exact values directly obtained form the charge density form factor in the $\mathrm{Sn}$ isotopic chain (for 

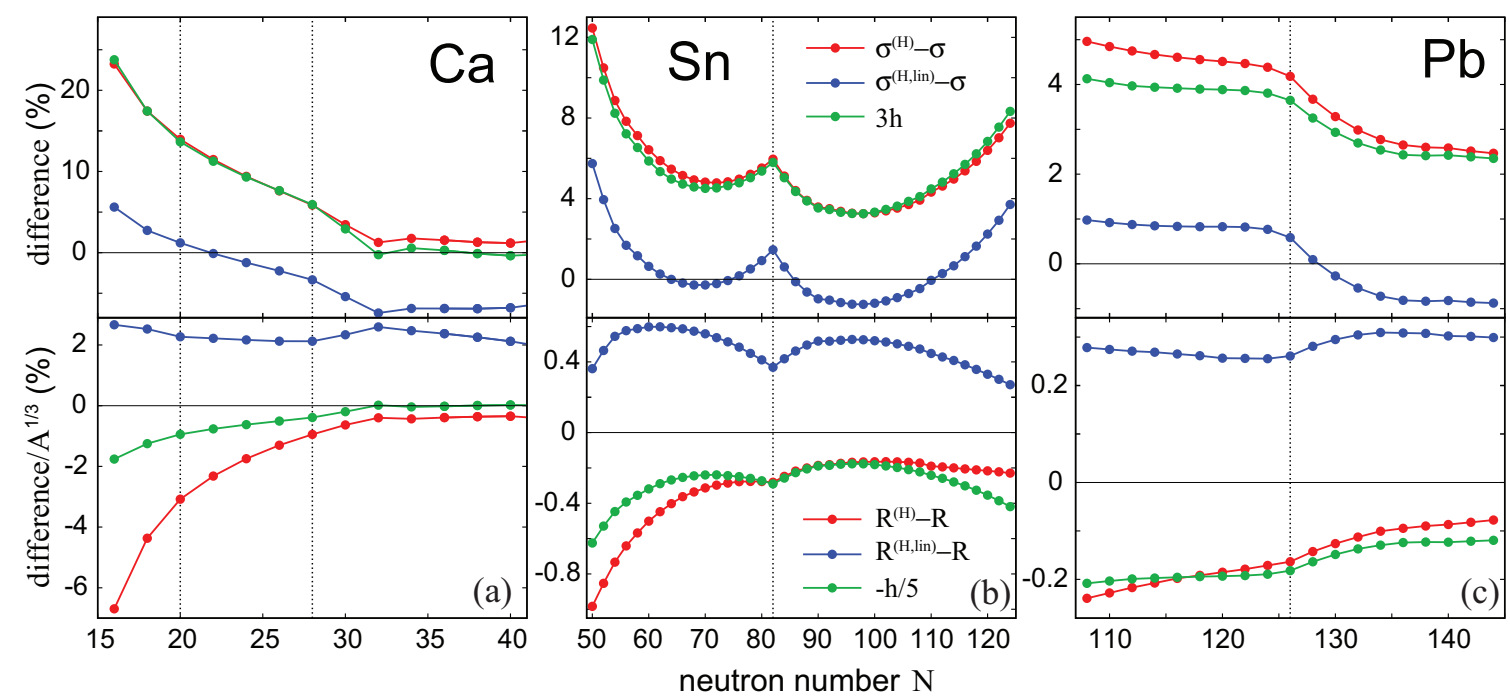

FIG. 4. Relative difference of Helm-model predictions (4)-(7) from the form factor values of $R$ (lower) and $\sigma$ (upper) for the isotopic chains of $\mathrm{Ca}(\mathrm{a}), \mathrm{Sn}(\mathrm{b})$, and $\mathrm{Pb}$ (c) computed with SV-min.

$\mathrm{Ca}$ and $\mathrm{Pb}$ chains, see Ref. [27]). The predictions based on $r_{2}$ and $r_{4}$ are fairly accurate. Indeed, for both $R$ and $\sigma$, the deviations of the Helm estimates from the form factor values are close to the computed uncertainties. Particularly good is the agreement for $R$ as the deviation between $R^{(\mathrm{H})}$ and $R$, around $0.02 \mathrm{fm}$ is smaller than the adopted error of diffraction radii $(0.04 \mathrm{fm})$. Interestingly, the linearized $\sigma^{(\mathrm{H}) \text {,lin }}$ performs exceptionally well except for the most proton-rich isotopes, in which the appreciable halo feature appears.

Figure 4 shows the relative differences (in \%) between form parameters $R$ and $\sigma$ and the Helm-model predictions for three different magic chains: $\mathrm{Ca}, \mathrm{Sn}$, and $\mathrm{Pb}$ [see Ref. [27] for the absolute differences in $\mathrm{fm}$, and for additional information on $\left.(\sigma / R)^{2}\right]$. It is seen that the quality of the Helm-model predictions improves significantly with increasing system size (see Ref. [39]). The density distributions of $\mathrm{Ca}$ isotopes are strongly impacted by surface effects and thus harder to describe by the simple Helm parametrization, while the $\mathrm{Pb}$ isotopes are volume dominated; hence, they are well approximated by the Helm model. But the general features observed before for the Sn chain remain: $R$ is better predicted than $\sigma$, and the linearized prediction for $\sigma$ performs unexpectedly well for all isotopic chains (though at different levels of overall quality).

Altogether, we see that the Helm-model analysis nicely corroborates the findings from statistical analysis. Figure 4 contains one more piece of information: It compares the differences with multiples of the halo parameter (3). It is interesting to see that the differences $R^{(\mathrm{H})}-R$ and $\sigma^{(\mathrm{H})}-\sigma$ are proportional to $h$. This observation is fairly consistent within the model. Zero halo means that the exact distribution is described fully by the Helm model. In this case, the differences would be zero. A mismatch leads to a finite halo and the same mismatch propagates to the predictions. This suggests a way to develop model-corrected predictions for $R$ and $\sigma$ from $r_{n}$ measurements. For a given isotopic chain, the halos follow regular trends which can be tracked in density-functional calculations. Namely, one can correct the Helm-model predictions by predicted halo values to obtain the form parameters $R$ and $\sigma$ in exotic nuclei from $r_{n}$ measurements with well-defined uncertainties that are well below those of the Helm model.

Conclusions. In this study, we assessed the impact of precise experimental determination of $\left\langle r^{4}\right\rangle$ on nuclear structure research. By means of statistical correlation analysis, we demonstrated that the diffraction radius $R$ and surface thickness $\sigma$ are well determined by $\left\langle r^{2}\right\rangle$ and $\left\langle r^{4}\right\rangle$, especially for heavy nuclei. Using the Helm model for the charge density, we could develop simple and robust relations for predicting $R$ and $\sigma$ from given $\left\langle r^{2}\right\rangle$ and $\left\langle r^{4}\right\rangle$. A higher predictive power is obtained when using $\left\langle r^{2}\right\rangle$ and $\left\langle r^{4}\right\rangle$ as input to fits of selfconsistent model and retrieving $R$ and $\sigma$ from them. This result was obtained by studying a broad selection of semimagic spherical nuclei. We expect that our conclusions apply for moderately deformed nuclei because it is a spherically averaged (monopole) charge density that is used in the analysis of the isotope shifts. The situation is less clear, however, for exotic topologies of charge density as, e.g., in nuclei with an appreciable spherical depression. In such cases, the Helm model needs to be modified to account for new effects [37,40].

It is to be noted that for the nuclei for which the charge density/form factor is experimentally known in the large range of $r$, the values of $r_{4}$ are well constrained by electron scattering data. In this context, we note that the values of $R$ and $\sigma$, and often also $r_{2}$, are determined from the charge form factor at low momentum transfer $q$, typically $q<6 / A^{1 / 3} \mathrm{fm}^{-1}$ [23].

Our findings are well aligned with the recent experimental progress $[1,5,15-18,41-43]$. We hope these results will provide a strong motivation for the next generation of precision experiments at radioactive beam facilities worldwide. Experimental determination of $\left\langle r^{4}\right\rangle$ would be extremely valuable for nuclei where the form factor data are not available. Since electron scattering experiments on unstable nuclei are highly 
demanding, if not impossible, precise measurements of atomic transitions would offer an alternative path to surface properties of unstable nuclei. Finally, as shown in Fig. 1, information on $\left\langle r^{4}\right\rangle$ would be very useful for better constraining current energy density functionals.
Acknowledgments. This material is based upon work supported by the US Department of Energy, Office of Science, Office of Nuclear Physics under Awards No. DE-SC0013365 (Michigan State University) and No. DE-SC0018083 (NUCLEI SciDAC-4 Collaboration).
[1] M. S. Safronova, D. Budker, D. DeMille, D. F. J. Kimball, A. Derevianko, and C. W. Clark, Rev. Mod. Phys. 90, 025008 (2018).

[2] J. C. Berengut, D. Budker, C. Delaunay, V. V. Flambaum, C. Frugiuele, E. Fuchs, C. Grojean, R. Harnik, R. Ozeri, G. Perez, and Y. Soreq, Phys. Rev. Lett. 120, 091801 (2018).

[3] Y. V. Stadnik, Phys. Rev. Lett. 120, 223202 (2018).

[4] C. Delaunay, C. Frugiuele, E. Fuchs, and Y. Soreq, Phys. Rev. D 96, 115002 (2017).

[5] R. F. Garcia Ruiz, M. L. Bissell, K. Blaum, A. Ekström, N. Frömmgen, G. Hagen, M. Hammen, K. Hebeler, J. D. Holt, G. R. Jansen et al., Nat. Phys. 12, 594 (2016).

[6] P. Campbell, I. Moore, and M. Pearson, Prog. Part. Nucl. Phys. 86, 127 (2016).

[7] M. Hammen, W. Nörtershäuser, D. L. Balabanski, M. L. Bissell, K. Blaum, I. Budincevic, B. Cheal, K. T. Flanagan, N. Frömmgen, G. Georgiev, C. Geppert, M. Kowalska, K. Kreim, A. Krieger, W. Nazarewicz, R. Neugart, G. Neyens, J. Papuga, P.-G. Reinhard, M. M. Rajabali, S. Schmidt, and D. T. Yordanov, Phys. Rev. Lett. 121, 102501 (2018).

[8] B. A. Marsh, T. Day Goodacre, S. Sels, Y. Tsunoda, B. Andel, A. N. Andreyev, N. A. Althubiti, D. Atanasov, A. E. Barzakh, J. Billowes et al., Nat. Phys. 14, 1163 (2018).

[9] C. Gorges, L. V. Rodríguez, D. L. Balabanski, M. L. Bissell, K. Blaum, B. Cheal, R. F. Garcia Ruiz, G. Georgiev, W. Gins, H. Heylen, A. Kanellakopoulos, S. Kaufmann, M. Kowalska, V. Lagaki, S. Lechner, B. Maaß, S. Malbrunot-Ettenauer, W. Nazarewicz, R. Neugart, G. Neyens, W. Nörtershäuser, P.-G. Reinhard, S. Sailer, R. Sánchez, S. Schmidt, L. Wehner, C. Wraith, L. Xie, Z. Y. Xu, X. F. Yang, and D. T. Yordanov, Phys. Rev. Lett. 122, 192502 (2019).

[10] A. J. Miller, K. Minamisono, A. Klose, D. Garand, C. Kujawa, J. D. Lantis, Y. Liu, B. Maaß, P. F. Mantica, W. Nazarewicz et al., Nat. Phys. 15, 432 (2019).

[11] C. Delaunay, R. Ozeri, G. Perez, and Y. Soreq, Phys. Rev. D 96, 093001 (2017).

[12] C. Frugiuele, E. Fuchs, G. Perez, and M. Schlaffer, Phys. Rev. D 96, 015011 (2017).

[13] V. V. Flambaum, A. J. Geddes, and A. V. Viatkina, Phys. Rev. A 97, 032510 (2018).

[14] K. Mikami, M. Tanaka, and Y. Yamamoto, Eur. Phys. J. C 77, 896 (2017).

[15] F. Gebert, Y. Wan, F. Wolf, C. N. Angstmann, J. C. Berengut, and P. O. Schmidt, Phys. Rev. Lett. 115, 053003 (2015).

[16] B. Braverman, A. Kawasaki, E. Pedrozo-Peñafiel, S. Colombo, C. Shu, Z. Li, E. Mendez, M. Yamoah, L. Salvi, D. Akamatsu, Y. Xiao, and V. Vuletić, Phys. Rev. Lett. 122, 223203 (2019).

[17] T. Manovitz, R. Shaniv, Y. Shapira, R. Ozeri, and N. Akerman, Phys. Rev. Lett. 123, 203001 (2019).

[18] A. Papoulia, B. G. Carlsson, and J. Ekman, Phys. Rev. A 94, 042502 (2016).
[19] J. Ekman, P. Jönsson, M. Godefroid, C. Nazé, G. Gaigalas, and J. Bieroń, Comput. Phys. Commun. 235, 433 (2019).

[20] B. K. Sahoo, A. R. Vernon, R. F. Garcia Ruiz, C. L. Binnersley, J. Billowes, M. L. Bissell, T. E. Cocolios, G. J. Farooq-Smith, K. T. Flanagan, W. Gins et al., New J. Phys. 22, 012001 (2020).

[21] W. King, Isotope Shifts in Atomic Spectra (Springer Science \& Business Media, Berlin, 2013), Vol. 11, p. 210.

[22] R. H. Helm, Phys. Rev. 104, 1466 (1956).

[23] J. Friedrich and N. Vögler, Nucl. Phys. A 373, 192 (1982).

[24] J. Friedrich and P.-G. Reinhard, Phys. Rev. C 33, 335 (1986).

[25] D. Andrae, Phys. Rep. 336, 413 (2000).

[26] M. Bender, P.-H. Heenen, and P.-G. Reinhard, Rev. Mod. Phys. 75, 121 (2003).

[27] See Supplemental Material at http://link.aps.org/supplemental/ 10.1103/PhysRevC.101.021301 for details on the form parameters of the nuclear charge distribution; supplemental figures illustrating the differences between Helm-model predictions and form parameters; and the Fy $(\Delta r, \mathrm{HFB})$ parameters.

[28] S. Mizutori, J. Dobaczewski, G. A. Lalazissis, W. Nazarewicz, and P.-G. Reinhard, Phys. Rev. C 61, 044326 (2000).

[29] P. Sarriguren, M. K. Gaidarov, E. M. deGuerra, and A. N. Antonov, Phys. Rev. C 76, 044322 (2007).

[30] N. Schunck and J. L. Egido, Phys. Rev. C 78, 064305 (2008)

[31] P. Klüpfel, P.-G. Reinhard, T. J. Bürvenich, and J. A. Maruhn, Phys. Rev. C 79, 034310 (2009).

[32] P.-G. Reinhard and W. Nazarewicz, Phys. Rev. C 95, 064328 (2017)

[33] P.-G. Reinhard, M. Bender, K. Rutz, and J. Maruhn, Z. Phys. A 358, 277 (1997).

[34] S. J. Krieger, P. Bonche, H. Flocard, P. Quentin, and M. S. Weiss, Nucl. Phys. A 517, 275 (1990).

[35] J. Erler, P. Klüpfel, and P.-G. Reinhard, Eur. Phys. J. A 37, 81 (2008).

[36] J. Dobaczewski, W. Nazarewicz, and P.-G. Reinhard, J. Phys. G 41, 074001 (2014).

[37] B. Schuetrumpf, W. Nazarewicz, and P.-G. Reinhard, Phys. Rev. C 96, 024306 (2017).

[38] P. D. Allison, Multiple Regression: A Primer (Sage, Thousand Oaks, CA, 1998).

[39] P.-G. Reinhard, M. Bender, W. Nazarewicz, and T. Vertse, Phys. Rev. C 73, 014309 (2006).

[40] J. Friedrich, N. Voegler, and P.-G. Reinhard, Nucl. Phys. A 459, 10 (1986).

[41] M. G. Kozlov, M. S. Safronova, J. R. Crespo López-Urrutia, and P. O. Schmidt, Rev. Mod. Phys. 90, 045005 (2018).

[42] C. A. Holliman, M. Fan, and A. M. Jayich, Phys. Rev. A 100, 062512 (2019).

[43] M. Fan, C. A. Holliman, A. L. Wang, and A. M. Jayich, Phys. Rev. Lett. 122, 223001 (2019). 